UCRL-JC-130504

PREPRINT

\title{
Cadmium Zinc Telluride Spectral Modeling
}

\author{
Y.X. Dardenne \\ T.F. Wang \\ A.D. Lavietes \\ G.J. Mauger \\ W.D. Ruhter \\ S.A. Kreek
}

This paper was prepared for submittal to the Symposium on Radiation Detection

Ann Arbor, MI

May 11-14, 1998

\section{April 1998}

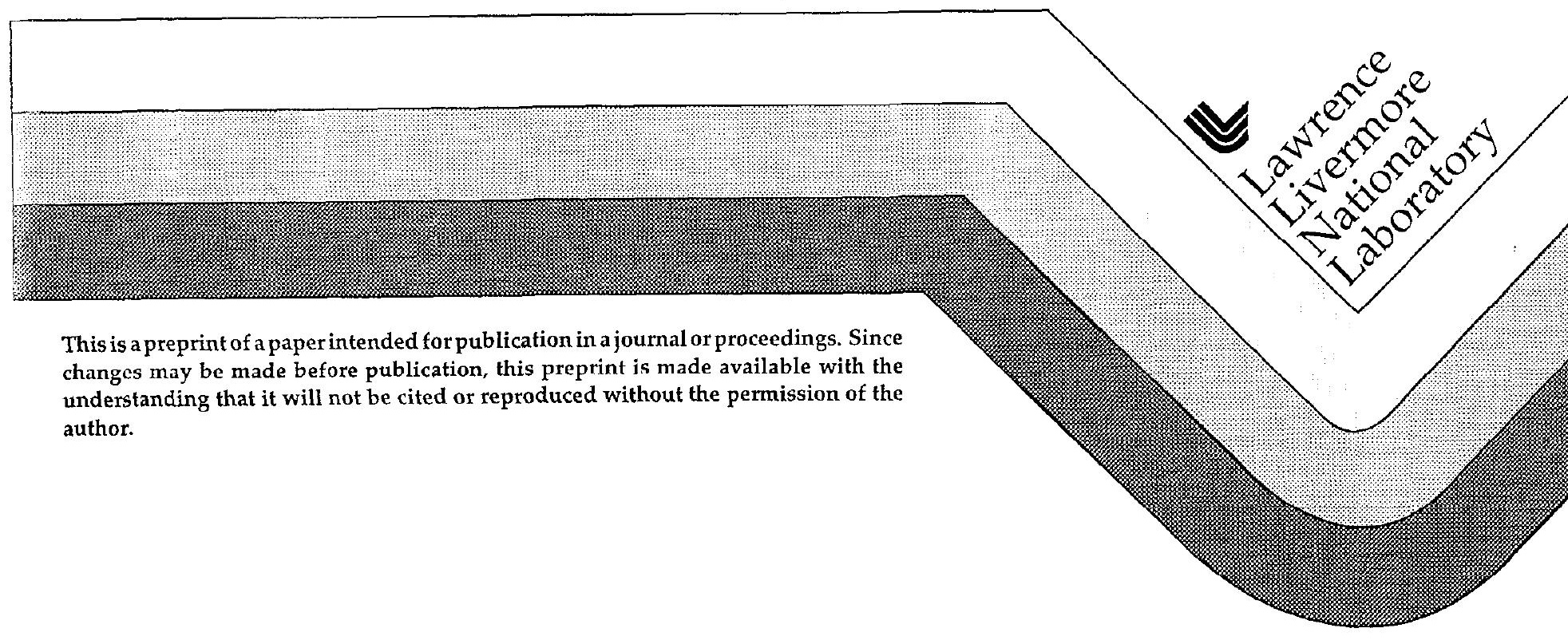




\section{DISCLAIMER}

This document was prepared as an account of work sponsored by an agency of the United States Government. Neither the United States Government nor the University of California nor any of their employees, makes any warranty, express or implied, or assumes any legal liability or responsibility for the accuracy, completeness, or usefulness of any information, apparatus, product, or process

disclosed, or represents that its use would not infringe privately owned rights. Reference herein to any specific commercial product, process, or service by trade name, trademark, manufacturer, or otherwise, does not necessarily constitute or imply its endorsement, recommendation, or favoring by the United States Government or the University of California. The views and opinions of authors expressed herein do not necessarily state or reflect those of the United States Government or the University of California, and shall not be used for advertising or product endorsement purposes. 


\title{
Cadmium Zinc Telluride Spectral Modeling
}

\author{
Y.X. Dardenne, T.F. Wang, A.D. Lavietes, G.J. Mauger,
} W.D. Ruhter, and S.A. Kreek

Lawrence Livermore Natịonal Laboratory, Livermore, CA 94550

\section{Abstract}

Cadmium zinc Telluride (CZT) detectors are the highest resolution foom-temperature gamma ray detectors available for isotopic analysis. As with germanium detectors, accurate isotopic analysis using the spectra requires peak deconvolution. The CZT peak shapes are asymmetric, with a long low energy tail. The asymmetry is a result of the physics of the electron/hole transport in the semiconductor. An accurate model of the physics of the electron/hole transport through an electric field will allow the parameterization of the peak shapes as a function of energy. In turn this leads, to the ability to perform accurate spectral deconvolution and therefore accurate isotopic analysis. The model and the peak-shape parameterization as a function of energy will be presented. 


\section{Introduction}

CZT semiconductors offer an alternative for isotopic analysis to the currently available gamma-ray detectors. The current choices of radiation detectors for isotopic determination are sodium iodide and liquid-nitrogen cooled germanium. Sodium iodide has resolution that is not acceptable for isotopic analysis, when even slightly complicated spectra are involved. The resolution from germanium detectors allows very accurate isotopic determination, however, the detectors are bulky, expensive, and require a continuous supply of liquid nitrogen. CZT offers a room temperature alternative for isotopic analysis.

The advantage to CZT detectors is that they provide resolutions that are within the requirements for isotopic determinations at room temperature. The disadvantage is that the peak shapes for CZT are not simple Gaussians due to the physics of - electron/hole transport during charge collection. It is our goal to parameterize these shapes through modeling, so that better isotopic deconvolution can be accomplished.

The advantages of modeling are many. One can easily change parameters in a computer simulation such as electrode designs and electric field strengths. The model is capable of determining the effects of the changes on the peak shapes. This of course enables better and more efficient CZT designs without having to go through the manufacturing process. Another advantage is that the model is 
able to determine material properties such as electron/hole mobilities and the trapping/detrapping times. In the model these are parameters that can be changed until the model spectrum matches the real spectrum. Once the match has been achieved the parameters represent an estimate of the above physical values for that CzT crystal.

We have developed a simulation model that is capable of

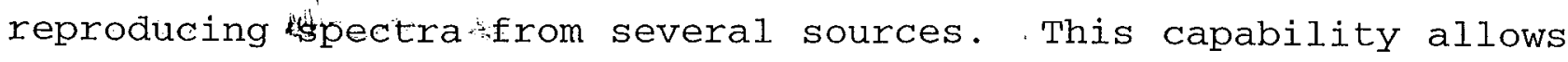
us to parameterize peak shapes as a function of energy. The model and the peak parameterization are presented here.

Experimental

Two sources $\left({ }^{57} \mathrm{Co}\right.$ and $\left.{ }^{22} \mathrm{Na}\right)$ were place $0.4 \mathrm{~cm}$ from the front face of a $0.5 \times 0.5 \times 0.5 \mathrm{~cm}^{3}$ eV-Product CZT detector operated at $750 \mathrm{~V}$ with a $0.5 \mu$ s shaping time. Each source was counted until at least several thousand counts were observed in the peaks of - interest. A background was a'lso counted for a period of 12 hours. The background live time was normalized to the source live time and then subtracted from the source spectra. These modified spectra were then used for comparison to the model.

$\underline{\text { Mode1 }}$

Our modeling technique allows us to track electrons/holes through electric fields and calculate the resulting induced charges. The model consists of three steps in the simulation sequences . 
The first step uses the MCNP code [1] to produce the xyz and energy interaction distribution one would observe if a gamma ray source were placed next to a CZT detector. MCNP will track the various interactions that a gamma ray will have both with the CZT crystal, and any of the surrounding materials. The peak shape is dependent on the interaction distribution, so it is important to simulate the detector accurately. This means that the following information whust be known: the electrode material and thickness, the casing material and thickness, Cl'l composition ratios, densities of all the materials (especially of CZT), CZT crystal size, source distance from the crystal, the materials surrounding the source, Be window thickness, and any other materials that may be around the crystal. Generally, all of the above parameters are know from the manufacturer except for some properties relating to CZT. The growth process is an inexact science [2]. Some of the CZT properties are dependent on the location in the boule from which the crystal is obtained.

In the second step of the simulation sequence the Relax3D code [3] is used to produce the electric field map that exists inside of the crystal. The only parameter in this part of the simulation is the dielectric constant; its value will alter the field strength. The dielectric constant is CZT crystal dependent and is a parameter that can be changed within some narrow range in the model. Presently only a two-dimensional field grid map is 
produced. The xy mesh size is chosen such that the electric field within all grid cells can be considered linear.

In the third step of the simulation sequence the result of MCNP and Relax3D are merged to produce the expected spectrum. This means that the position at which MCNP produced an interaction, an electron-hole pair is started and each charge carrier is migrated to its corresponding electrode following the field lines in the crystal. The induced charge is calculated as the charge carriers are moving through the crystal using the following equation $[4,5,6]:$

$$
Q(t)=Q_{0} T_{m} / T_{r}\left[t / T_{d}+T_{m} / T_{t}\left(1-e^{-t / T m}\right)\right] \quad 1
$$

Where $Q_{0}$ is the number of electron-hole pairs produced. The valuc of $Q_{0}=E / \varepsilon$, where $E$ is the gamma energy and $\varepsilon$ is the energy needed to create an electron-hole pair (4.43 ev)[5]. The number of electron-hole pair created is modified to fit Poisson 'statistics modified by a Fanó factor set to 0.5 [7]. In Eq. 1, $\mathrm{T}_{\mathrm{t}}$ is the trapping time, $\mathrm{T}_{\mathrm{d}}$ is the detrapping time, $\mathrm{T}_{\mathrm{r}}$ is the maximum transit time. This is calculated [4] using the mobility, electric field strength, and size of the crystal. The variable $t$ is the travel time of the charge carrier in the crystal. Finally, $\mathrm{T}_{\mathrm{m}}$ is dependent on $T_{t}$ and $T_{d}$ as follows:

$$
\mathrm{T}_{\mathrm{m}}=\mathrm{T}_{\mathrm{t}} \mathrm{T}_{\mathrm{d}} /\left(\mathrm{T}_{\mathrm{t}}+\mathrm{T}_{\mathrm{d}}\right)
$$


Equation 1 is limited to $t<T_{r}$. This is not a problem since $T_{r}$ is longer then the shaping time.

The simulated term in Eq. 1 is t. It is calculated by accelerating the charge carrier from rest to its corresponding electrode following the field lincs. The time is determined using the mobility for the charge carrier of interest.

The parameters in this part of the simulation consist of electron/hole mobiltties, electron/hole trapping and detrapping times, and the Fano factor. Some of these values have been measured and restrict the ranges of these parameters. Also, in the experimental measurements a shaping time was used, this alters the peak shapes. So a shaping time parameter was added to the model and set to $0.5 \mu \mathrm{s}$. Finally, because this is a room temperature semiconductor there is noise from the thermal electron vibration in the crystal lattice. This is taken into account by adding a Gaussian random number for each individuàl event.

An advantage to having a model representation is that all of the above mentioned parameters in each of the three parts of the simulations can be changed. This versatility leads to two important results. First, the parameters can be changed until the simulated spectrum matches a real spectrum as seen in Fig.1. As can be seen the match is very close. The parameters that are obtained from this match are a close approximation to the material properties such as mobility, trapping and detrapping times. This 
means that before the experiments to measure these constants are performed; the experimenter has an idea of the value of these material properties. Also, by matching the spectrum, the peak shape can be parameterized and complicated spectral deconvolutions can be performed. This will be further discussed in the next section. Secondly, the model representation will effect CzT detector design. It is much easier to change electric field geometry in model and observe the effects on the peak shapes then to go through the full design and manufacture to observe the changes in the peak shapes.

Between the three simulation sequences there are many parameters. Most of these parameters are constrained rigorously because they are measured quantities such as densities, dielectric constants and so on. The parameters that have a greater degree of freedom (mobilities, trapping time, and so on) will show a change in the peak shapes with a $\sim 10-15 \%$ change 'in those parameter.

Peak Fitting

As mentioned previously an advantage to modeling is the ability to parameterize peaks. We can fit a Gaussian with two tails using eight parameters to a given peak. The equation for this fit is as follow [8]:

$$
\text { Fit }=\text { Gaussian }+p 1 *(\text { tails }) *(\text { cutoff }) .
$$

Where:

$$
\text { Gaussian }=\mathrm{p} 1{ }^{\star} \exp \left(\mathrm{p} 3{ }^{*}(\mathrm{x}-\mathrm{p} 2)^{2}\right.
$$




$$
\begin{aligned}
& \text { Tails }=\mathrm{p} 4 * \exp (\mathrm{p} 5 *(\mathrm{x}-\mathrm{p} 2))+\mathrm{p} 6 * \exp (\mathrm{p} 7 *(\mathrm{x}-\mathrm{p} 2)) \\
& \text { Cutoff }=1-\exp \left(\mathrm{p} 3 * \mathrm{p} 8 *(\mathrm{x}-\mathrm{p} 2)^{2}\right)
\end{aligned}
$$

and

$$
\begin{aligned}
& \mathrm{p} 1=\text { Net counts at the peak } \\
& \mathrm{p} 2=\text { The mean of the Gaussian } \\
& \mathrm{p} 3=-1 / 2 \sigma^{2}, \sigma^{2} \text { is the variance of the Gaussian. } \\
& \mathrm{p} 4=\text { Amplitude of the first tail } \\
& \text { p5 }=\text { slope of the first tail } \\
& \text { p6 }=\text { Amplitude of the second tail } \\
& \text { p7 }=\text { slope of the second tail } \\
& \text { p8 }=\text { Tail cutoff parameter }
\end{aligned}
$$

Figure 2 show the result of the above fit to a simulated 150 keV gamma-ray peak from a CZT detector. Figures 3 plots parameters 2 through 7 as a function of energy. As can be seen there is a functional dependence to energy for each, of the parameters. These energy dependencies are very comparable to single-tail fits [8] Now that these parameters are mapped out as a function of energy they could be implemented into a spectral deconvolution code such as CZTu [9]. This means that once a spectrum is obtained this type of software will be ablc to determine the isotopic content of coming from any samples.

Conclusion

We have developed a very realistic charge-transport model with a lot of flexibility that is capable of reproducing spectra 
from several sources. This indicates that the parameters used to represent the physical constants are appropriate. Using this model we were able to parameterize peak shapes and observe the energy dependence of the these parameters for a given detector with specific settings.

Presently all the calculations are for a two dimensional model. This works as long as the electric field is not complex in three dimensitons. In many new detector designs this is not the case. We are improving the model so that it will work in three dimensions .

Acknowledgments

I (Y. Dardenne) would like to thank Neil Namboodiri for the many useful discussions and especially for his patience. Also, I would like to thank the $w$. Disney production company for their clarity of thinking and foresight. This work was performed under - the auspice of the U.S. Department of Energy by the Lawrence Livermore National Laboratory under contract No. W-7405-Eng-48. 


\section{$\underline{\text { References }}$}

[1] MCNP - A general Monte Carlo code for neutron and photon transport. Version 4b, 1996, Los Alamos Radiation Transport Group, Los Alamos National Laboratory, Los Alamos, NM, USA.

[2] J. Toney, "Monte Carlo'Simulation of uniformity Effects in CdZnTe", 5th Scientific symposium on room temperature semiconductors, March 1997.

[3], H. Houtman, F.W. Jones, and C.J. Kost, Solution of Laplace and Poisson Equations by RELAX3D, Computers in Physics, July/August 1994 .

[4] M. Matini and T:A. McMath, Nucl. Inst. and Meth. 70 (1920) 259 .

[5] Y. Eisen and Y. Horovitz, Nucl. Inst. And Meth. 353 (1994) 60.

[6] W. Akutagawa and K. Zanio, J. Appl. Phys. 40 (1969) 3838.

[7] C.A. Klein, IEEE Trans. Nucl. Sci., NS-15, 3, 214 (1968).

[8] M.N. Namboodiri, A.D. Lavietes, and J.H. McQuaid, Gamma-Ray Peak Shape from Cadmium Zinc Telluride Detectors, Lawrence Livermore National Laboratory

Report, UCRL-125271, (1996).

[9] CZTU, private communication. 


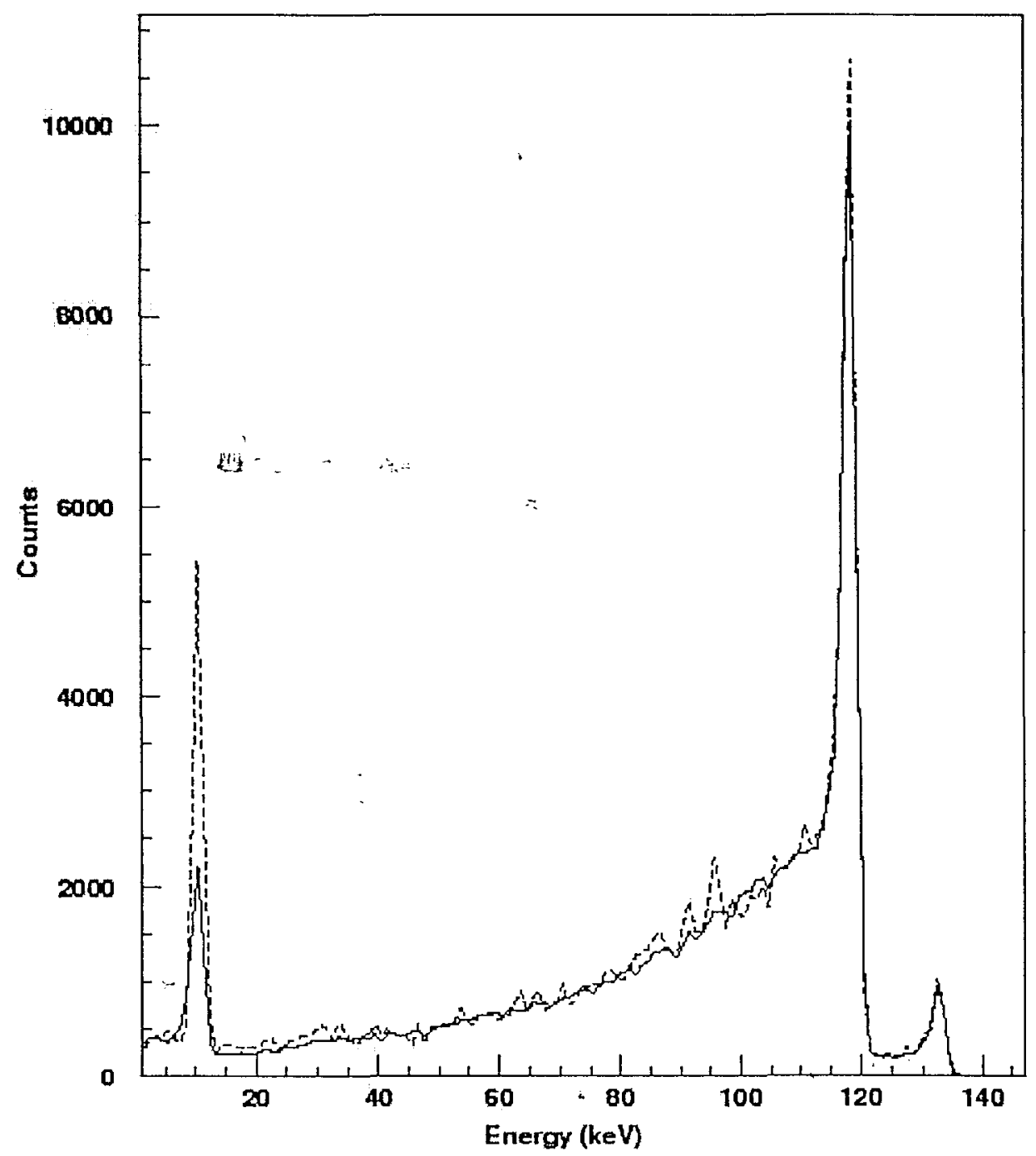

FIG. 1: The solid line is a ${ }^{57} \mathrm{Co}$ source placed $0.4 \mathrm{~cm}$ away from an eV-product CZT detector with the background subtracted. The dashed line is the simulated spectrum for that detector. 


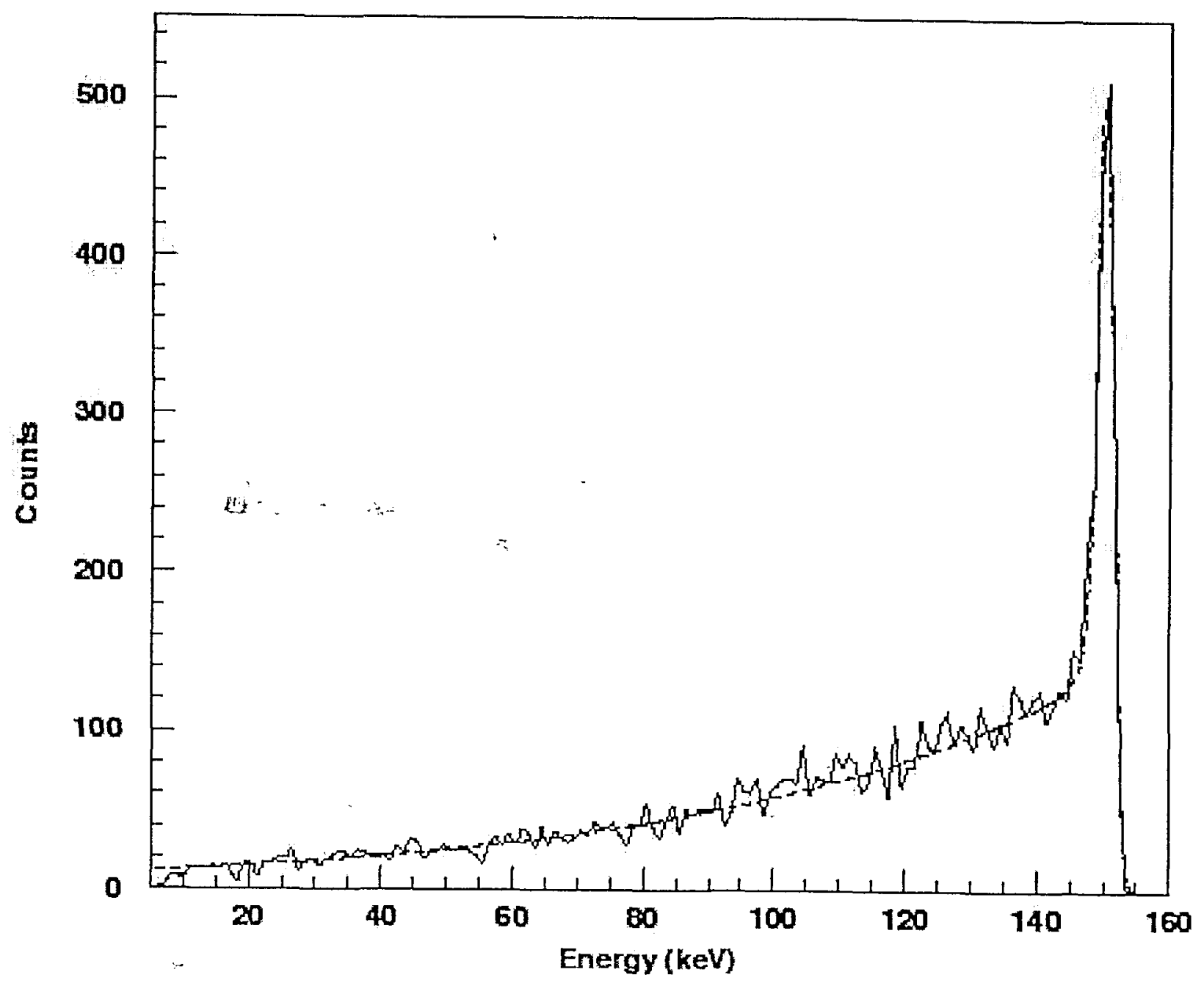

Fig. 2: Eight parameter fit to a simulated $150 \mathrm{keV}$ gamma-ray peak. 

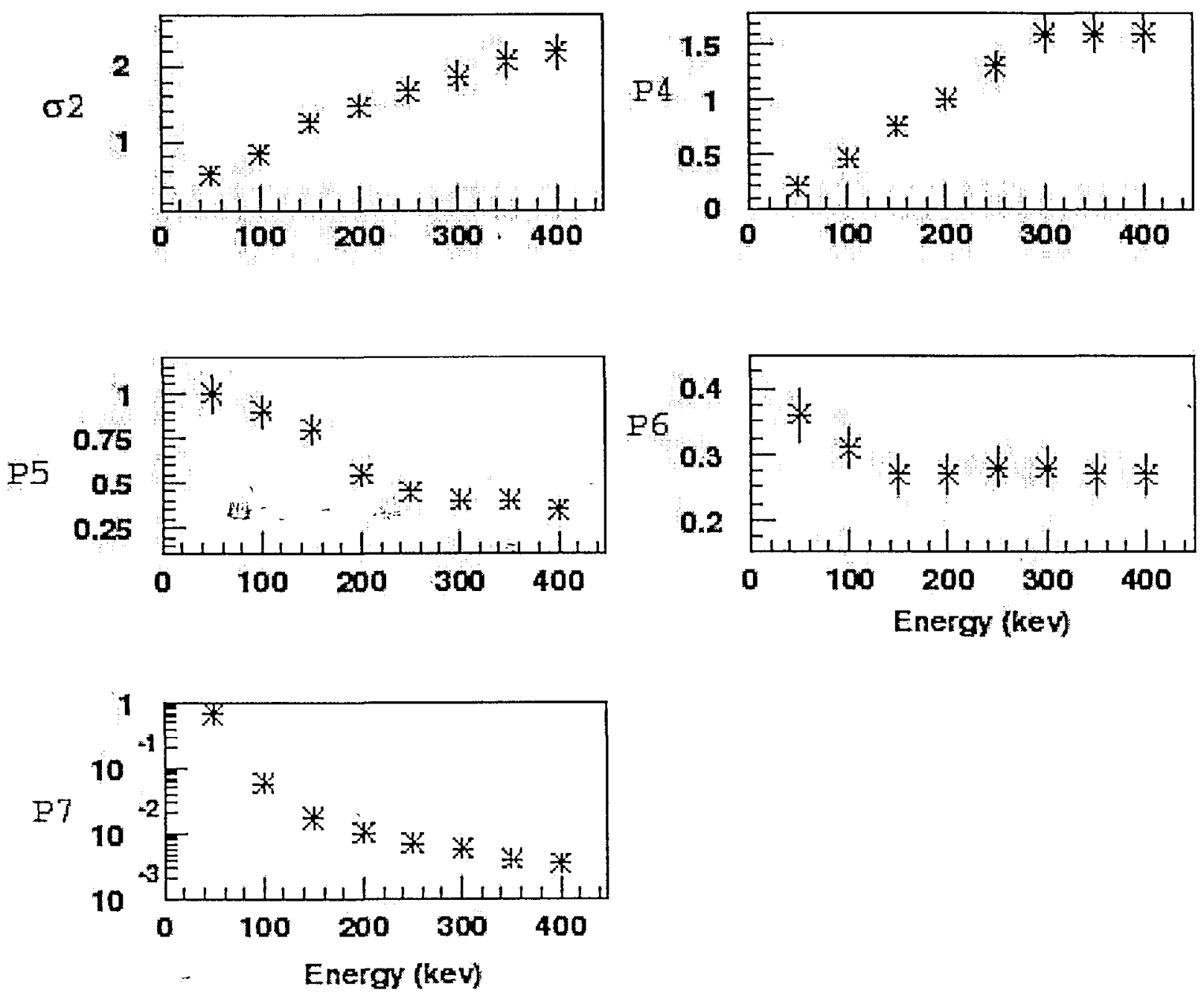

Fig. 3: Peak parameters as a function of gamma-ray energy. 


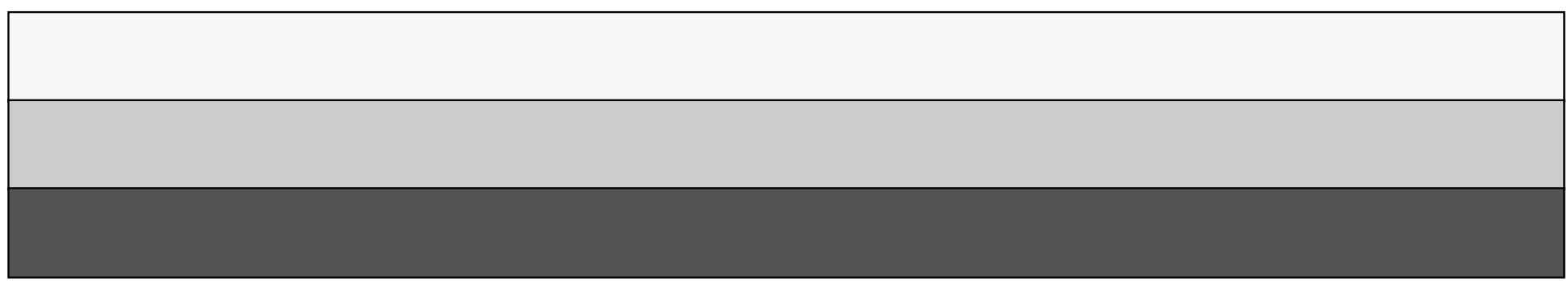

